genheit abschließend zu bewerten. Oftmals - und das machen die Beispiele deutlich kehren sich kurzfristige Verbesserungen ins Gegenteil um.

Sehr schön arbeiten die Verfasser die verschiedenen Konfliktfelder heraus, auch wenn bisweilen eine an sich logische Argumentation durch polemischen Stil verliert. Die Forderungen und Lösungsvorschläge, die gezielt an den dargestellten Problemen ansetzen, werden allerdings schwer realisierbar sein, da gerade die USA, ${ }^{4}$ und bedenkt man die Anlehnung Großbritanniens und der Bundesrepublik Deutschland an deren Haltung, vielfach konträrer Auffassung sind. So dürfte die Forderung nach einer umfassenden Quotenerhöhung fehlschlagen. ${ }^{5}$ Andere Vorschläge, so zu einer Demokratisierung der IWF-Tätigkeit, sind, so einleuchtend sie zunächst erscheinen, doch auf etwaige Auswirkungen zu untersuchen. Die Autoren befürworten etwa, daß bei Aushandlung der Bedingungen für einen Kredit die gesellschaftlichen Gruppen eines Landes berücksichtigt werden sollen. Dies stellt aber zweifelsohne einen Eingriff in die Souveränität der jeweiligen Regierung dar. So wünschenswert dies zwar, und auch das machen die Länderbeispiele deutlich, manchmal wäre, so kann es doch nicht Aufgabe einer Wirtschaftsorganisation sein, auf den innerstaatlichen Entscheidungsprozeß dergestalt Einfluß zu nehmen. Außerdem würden dadurch politische Faktoren (Zustimmung der Gewerkschaft notwendig? Zustimmung der Contras notwendig?) verstärkt Zutritt in den Einflußbereich des IWF haben, etwas, was angesichts der Forderung nach einer politisch neutralen Organisation bedenklich erscheint.

Es wäre wünschenswert, wenn dieses preisgünstige Buch einen breiten Leserkreis finden würde, da es zum einen für den Allgemeininteressierten eine gute Darstellung der Verschuldungsprobleme beinhaltet, zum anderen für den mit der Problematik vertrauten Leser umfassendes Material enthält und Denkanstöße liefert.

Wolfgang Engshuber

Hans-Joachim Konrad (Hrsg.)

Grundrechtsschutz und Verwaltungsverfahren - Internationaler Menschenrechtsschutz

Duncker und Humblot, Berlin, Schriften zum Offentlichen Recht, Band 484, 1985, 315 S., DM 88,-

Der vorliegende Band faßt die Referate zusammen, die anläßlich der 23. Tagung der wissenschaftlichen Mitarbeiter der Fachrichtung "Offentliches Recht" im Jahre 1983 in Berlin gehalten worden sind. Nach einer kurzen Einleitung des Herausgebers folgen die

4 May, Bernhard: Die Entwicklungspolitik der Reagan-Administration, in: Aus Politik und Zeitgeschichte, Beilage zur Wochenzeitung Das Parlament, B 49/84, 8. Dez. 84, S. 3 ff. (S. 14 f.).

5 Die 8. Quotenerhöhung 1983 war in den USA lange Zeit äußerst umstritten. Zudem ziehen sich die USA aus internationalen Organisationen mehr und mehr zurück und verlagern ihre Hilfe auf bilaterale Abkommen. 
Vorträge, die etwa den Stand von Mitte 1984 widerspiegeln, in der Reihenfolge, wie sie gehalten worden sind. Dem entsprechen die beiden, nebeneinander stehenden Themenblöcke.

"Grundrechtsschutz und Verwaltungsverfahren" eröffnet mit Ausführungen von Rainer Pitschas zum Thema: "Der Verwaltungsstaat in der Krise: Vom Wandel des Verwaltungsverfahrens" (S. 23). Ausgehend von den Ansätzen der "Verfassungsbezüge des Verwaltungsverfahrens" und des "Verwaltungsverfahrens als Modell kommunikativer Rechtssetzung und Zweckkonkretisierung" diskutiert er Leistung und Defizite der Verwaltungsverfahrensgesetze. Solche - aus dem Strafprozeßrecht geläufige - Themen münden regelmäßig eher in anspruchsvolle Programme als in konkrete Ergebnisse, weil sie die Besonderheiten einzelner Materien in tatsächlicher wie rechtlicher Hinsicht nicht in das Blickfeld aufnehmen. Zudem leidet die Diskussion gerade in Deutschland daran, daß Verfahrensgrundsätze überwiegend ergebnisorientiert diskutiert werden. Auch das gerechteste Verwaltungs- oder Gerichtsverfahren garantiert allerdings noch längst kein gerechtes Ergebnis. Auch die gerechteste Fußballregel garantiert nicht, daß stets die bessere Mannschaft gewinnt. Vielmehr ist Verfahrensgerechtigkeit ein eigenes Ziel mit eigenem Wert, der keineswegs auf das bloß "formelle" reduziert erscheint. Hier vermag ein Blick auf Verfassung und Recht in Übersee wesentliche Anregungen zu vermitteln.

Ergänzend berichtet Hannes Tretter aus österreichischer Sicht über "Verwaltungsverfahrensrechtliche Aspekte der Grundrechtsgewährleistung" (S. 63). Er kritisiert das Grundrechtskonzept des Osterreichischen Verfassungsgerichtshofes, das bei einem "formalistisch-negatorischen Verständnis" stehengeblieben sei. Demgegenüber fordert er eine verstärkte Bemühung um objektive Grundrechtsdimensionen, um so deren Verfahrensbezug verstärkt zur Geltung bringen zu können.

$\mathrm{Da}$ solche Ansätze möglicherweise schon sehr weit gegriffen sind, zeigt die folgende Serie über "Asylverfahren und Grundrechtsgewährleistung" von Michael Kilian (S. 71), "Asylrecht und Asylverfahren in Österreich" von Stefan Rosenmayr (S. 113) sowie "Asylgewährung als Grundrechtsschutz - Das Asylverfahren in der Schweiz" von Walter Kälin (S. 161). Konkrete Ansätze für einzelne Sachgebiete und einzelne Grundrechte bieten hier die Chance konkreter Ergebnisse. Während Kilian die Geschichte des deutschen Asylgrundrechts als Geschichte seiner Einschränkungsversuche sieht (S. 78), hält er die verfahrensrechtlichen Restriktionen durch immer neue "Beschleunigungsnovellen" doch im wesentlichen für grundrechtskonform. Das zentrale Problem ist für ihn eher die mangelnde tatsächliche Aufnahmebereitschaft der Bundesrepublik als ein Mangel im geltenden Recht. Rosenmayr erläutert umfassend das Asylrecht Osterreichs, zu welchem Untersuchungen bislang eher Mangelware sind. Ansatzweise schimmert in seinen Ausführungen der Gedanke durch, daß die österreichische Rechtsordnung einen Anspruch politisch Verfolgter auf Anerkennung als Flüchtling und einen Anspruch des Anerkannten auf Aufenthalt anerkennt (S. 135). Damit hat Osterreich wesentliche Elemente des Rechts auf Asyl positiviert, und zwar mit Gesetzesrang. Kälin muß demgegenüber vom Fehlen eines solchen Rechts in der Schweiz ausgehen. Die dortige Rechtsordnung kennt nur einen Anspruch auf Verfahrensdurchführung, als formelle Rechtspositionen. Wie 
hier die Abhandlung aus einzelnen Grundrechten - insbesondere Menschenwürde, due process und rechtliches Gehör - Elemente eines rechtsstaatlichen Asylverfahrens begründet, ist an konziser Argumentation, klaren Ergebnissen und Weite der Perspektive eine Meisterleistung. In klarer Erkenntnis der Reichweite wie der Grenzen seiner Auffassung wird keine allgemeine Lehre begründet, sondern eine höchst besondere, dafür aber weiterführende. Hier weist sich, wo und wie "bereichsspezifisch" weiterzudenken ist und wo nicht.

Der 2. Teil ist dem völkerrechtlichen Menschenrechtsschutz gewidmet. Michael Sachs untersucht "Begrenzungen des Unterscheidungsverbotes nach Art. 14 EMRK" (S. 183). Seine bisweilen kasuistisch-einzelfallorientiert anmutende Argumentation verstärkt den von ihm selbst abgelehnten Eindruck, daß Gleichheitsrechte als Differenzierungsverbote aus besonderen Gründen anzusehen sind, also Begründungsgebote oder -verbote für Ungleichbehandlungen darstellen. In diesem Sinne würde auch Art. 14 EMRK wesentlich an Geltungsinhalt gewinnen. Dietrich Murswiek erörtert "Die Pflicht des Staates zum Schutz vor Eingriffen Dritter nach der EMRK" (S. 213). Ausgehend vom Fall Young, James and Webster entnimmt er allen Garantien der Konvention eine zusätzliche Dimension: Die Pflicht des Staates, durch Gesetze Grundrechtseingriffen Dritter vorzubeugen. Staatliche Zulassung privater Eingriffe sei selbst ein Eingriff. Diese Ansicht (s. schon Schwabe, Die sogenannte Drittwirkung der Grundrechte, 1971) weist allerdings grundrechtsdogmatische Probleme auf. Freiheiten realisieren sich im Verhalten der Menschen untereinander. Dabei kommt dem Staat eine vom Bürger durchaus verschiedene Rechtsstellung zu. Sollen auch privatrechtliche Beziehungen grundrechtlich geprägt sein, so würde eine derartige - notwendig institutionelle - Ausgestaltung allerdings in inhaltliche Abstimmungsschwierigkeiten geraten. Privates Handeln ist eben Freiheitsausübung und nicht notwendig Kompetenzausübung, wie es staatliche Maßnahmen notwendig sind. Soll der "eingreifende" Private nicht als Beliehener mit besonderen Kompetenzen qualifiziert werden - eine Sicht, die seiner eigenen Grundrechtsausübung kaum entspräche -, so kommt jede Ausgestaltung privater Rechtsbeziehungen an dem Umstand nicht vorbei: Abzugrenzen sind nicht staatliche Kompetenz und Bürgerfreiheit, sondern letztere unter mehreren Trägern. Damit wäre eine solche "Horizontaldimension" von der Staat-Bürger-Dimension notwendig verschieden. Nur: Wie sie dann sein soll, erfährt durch die Garantien der EMRK keine hinreichend deutliche Vorprägung. Damit bleiben aber die Maßstäbe der Horizontalwirkung unklar, sie können den Grundrechten nicht "entnommen" werden. Deutlich erkennt Murswiek diese Fragestellungen am Beispiel der Vertragsfreiheit (S. 230 f.), ohne allerdings daraufhin sein Konzept neu zu überprüfen. Letztlich gelangt er so zu einer - von ihm ausdrücklich (S. 217) offengelassenen - Drittwirkung der Grundrechte, ohne das Phänomen bei diesem - zutreffenden - Namen nennen zu wollen. Diese Drittwirkungsdiskussion ist allerdings längst - ohne hinlängliche Gründe für ihre Bejahung - geführt worden. So zutreffend Kommission und Gerichtshof den Fall Young u. a. entschieden haben mögen: Verallgemeinern lassen sich ihre - kaum begründeten - Ansichten nicht.

"Regionaler Menschenrechtsschutz im interkontinentalen Vergleich" ist das Thema von 
Philip Kunig (S. 243), der Gemeinsamkeiten und Unterschiede zwischen der Amerikanischen, Afrikanischen und Europäischen Menschenrechtskonvention abhandelt. Seine überaus kenntnisreichen Ausführungen machen deutlich, daß in den einzelnen Kontinenten der Menschenrechtsschutz einen völlig unterschiedlichen Stellenwert einnimmt. Während hier Europa und Amerika noch annäherungsweise vergleichbar sind, fällt Afrika aus dem - hier behandelten - Rahmen. Das gilt für die Völkerrechtstradition, die Rechtstradition wie auch hinsichtlich der Bedürfnisse der Menschen und Völker (prägnanter Uberblick S. 252 ff.). Ist in Europa und Amerika die Staatsgewalt monopolisiert und zentralisiert, so muß sie in Afrika oft um ihre Behauptung erst kämpfen. Sie ist entweder Spielball antagonistischer Stammesinteressen oder Beute der gerade stärksten Macht - meist des Militärs -; sie zeichnet sich durch das regelmäßige Fehlen staatstragender Volksschichten und eine of $t$ nur geringe Reichweite aus. Konkurrierende, lokale oder intermediäre Mächte sind "vor Ort" wesentlich bedeutsamer als die weit entfernte Regierung. Gegen eine solche Staatsgewalt reichen Grundrechte nicht sehr weit; sie können nicht weit reichen, sofern die tatsächlichen Einwirkungen solcher Staatsgewalt oft sehr begrenzt sind und die wirklichen, die Menschen treffenden Entscheidungen von anderen gefällt werden. Zudem haben die meisten Bürger größere Sorgen als die Verwirklichung von Freiheit und Gleichheit. Die Grundrechte auf Nahrung, Kleidung und Wohnung sind wichtiger. So werden Grundrechte im europäisch-amerikanischen Sinne eher zu Binnennormen der Machteliten in den Städten. Hieraus entsteht aber auch ihr Dilemma: Sind es diese Eliten, die zugleich um die Staatsmacht kämpfen - ein Kampf, in welchem die Staatsgewalt ihrerseits auch instrumentalisiert wird -, so steht und fällt Grundrechtsschutz mit der Stabilität des Staates: Je ernster die Lage ist und je dringender Grundrechtsschutz wird, desto weniger kann er noch durchgesetzt werden. Notstandsvorbehalte und Staatsnotrechte sind dann Existenzfragen der Staatsbehauptung. Die Bestrafung tatsächlicher oder vermeintlicher Gegner ist dann nicht nur Kriminalpolitik, sondern staatliche Selbstverteidigung. Solche Selbsterhaltungsbestrebungen der an der Macht Befindlichen durch Grundrechte zu zügeln, ist angesichts der vorhandenen Rahmenbedingungen ein überaus anspruchsvolles Unterfangen - nicht nur des Völkerrechts. Abschließend erörtert Wolfgang Benedek "Das Recht auf Entwicklung in universeller Sicht und im Rahmen des afrikanischen Menschenrechtsschutzes" (S. 275). Dabei geht er von der Parallele in der Entwicklung der Forderung nach einer Neuen Weltwirtschaftsordnung einerseits und dem Recht auf Entwicklung andererseits aus. Daß dieses Recht vielfach postuliert wird, hat allerdings noch nicht zu seiner inhaltlichen Klärung beigetragen. Sicherlich steht es im Kontext vielfältiger schon vorhandener Menschenrechte wie etwa Recht auf Leben, Gesundheit, körperliche Unversehrtheit. Aber es geht doch in mehreren entscheidenden Punkten darüber hinaus. "Entwicklung" ist mehr als bloße Hilfssendungen aus Europa oder Amerika. Sie ist vielmehr ein komplexer Prozeß, der nur von den betroffenen Menschen selbst unter Mitwirkung des eigenen Staates und ausländischer Helfer bzw. Hilfe vollzogen werden kann. In einem solchen, überaus vielschichtigen Prozeß helfen subjektive Rechte des Einzelnen kaum weiter. Worauf auch sollten sie sich richten? Wie sollten sie von den Staaten des Nordens erfüllt werden, ohne 
in die Kompetenzen der jeweiligen Entwicklungsländer einzugreifen? Was sollen die Anspruchsgegner überhaupt tun? Ein so komplexes Geflecht kann nicht mit einem neuen Menschenrecht aufgelöst werden. Benedek ist dann auch skeptisch (S. $307 \mathrm{ff}$.) und will wenn überhaupt - die Entwicklungsländer selbst als Treuhänder der Entwicklungsansprüche ihrer Bürger auftreten lassen. Angesichts der bisherigen Praxis stellt sich dann allerdings die Frage, ob damit nicht die Erfüllbarkeit des Anspruchs endgültig vereitelt wird. Das gilt um so mehr, als jede Hilfe aus dem Norden, bevor sie bei den Menschen in Afrika ankommt, notwendig eine oder mehrere Qualitätsänderungen durchmacht: Ein Dollarbetrag schafft in keinem Dorf einen neuen Brunnen oder eine Meerwasserentsalzung. Hier sind Betroffene, vorhandene Administration und subsidiäre personelle Hilfe von außen erforderlich. Jedes Recht auf Entwicklung bleibt so notwendig eine leere Hülse: Menschen oder Gemeinwesen können sich entwickeln, aber nicht entwickelt werden. Entwicklungsvölkerrecht ist ein Prinzip jedes zukünftigen wie auch des geltenden Völkerrechts. Hierfür Instrumente zu entwickeln, sind Rechtsordnungen und Rechtswissenschaft aufgerufen. Das Recht auf Entwicklung gehört - als subjektives Recht von Menschen oder Staaten - nicht dazu.

Der vorliegende Band sucht zu neueren Grundrechtsproblemen zum Teil neue Antworten. Gerade die vergleichenden Abhandlungen stellen einen wesentlichen Fortschritt für die deutsche Rechtswissenschaft dar. Zeigt sie sich gerade in Grundrechtsfragen oft überaus introvertiert, so lassen sich hier einem Vergleich neue Perspektiven abgewinnen, die den Horizont zu erweitern vermögen. Und das war eine wesentliche Aufgabe der Tagung.

Christoph Gusy

\section{Günther Rusch}

Die verhinderte Mitsprache - Aspekte zur Sprachpolitik in Ghana, Togo und Obervolta Arbeiten aus dem Institut für Afrika-Kunde; Bd. 47, Hamburg 1984

Schon der Titel des Buches gibt Auskunft über die Einschätzung des Autors bezüglich des behandelten Themas, ist er doch Ausdruck dafür, daß die Bevölkerung - wie Rusch es formuliert - in Ghana, Togo und Obervolta bei den augenblicklichen sprachpolitischen Gegebenheiten "nur schwerlich die Kenntnisse und Informationen erhält, um den neuen Staat oder zumindest die regionale sozio-ökonomische Entwicklung bewußt mitzutragen" (p. 4). Was Rusch hier auf drei Länder bezieht, hat in gleicher oder ähnlicher Form Gültigkeit für die Mehrheit der schwarzafrikanischen Staaten.

Ursache des Problems ist die Tatsache, daß die Mehrzahl der afrikanischen Staaten eine starke linguistische Zersplitterung aufweist, daß also in jedem Land eine Vielzahl von Sprachen Verwendung findet. Für die drei untersuchten Länder können (nach Dalby) nicht weniger als 127 einheimische Sprachen und Dialekte aufgelistet werden. Daß da- 